\title{
Intra-arterial thrombolysis in basilar artery occlusions combination of intra- arterial thrombolytics and Gp IIb/IIla inhibitors in basilar artery thrombosis
}

\author{
S. B. Gaikwad, M. V. Padma ${ }^{1}$, E. J. Moses, Kanika Sethi, M. Tripathi ${ }^{1}$, R. Bhatia ${ }^{1}$, K. $\operatorname{Prasad}^{1}$, N. K. Mishra \\ Departments of Neuroradiology, ${ }^{1}$ and Neurology, All India Institute of Medical Sciences, Ansari Nagar, \\ New Delhi-110 029, India
}

\begin{abstract}
Address for correspondence:
Dr. Shailesh B. Gaikwad

Department of Neuroradiology, All

India Institute of Medical Sciences,

Ansari Nagar, New Delhi -1 10029 , India.

E-mail: sgaikwad_63@yahoo.com
\end{abstract}

DOI: $10.4103 / 0028-3886.53291$

\begin{abstract}
Basilar artery thrombosis has high morbidity and mortality. Though intra-arterial thrombolytics have proven efficacy in the treatment of acute basilar artery occlusion, the elevation of procoagulant factors in the blood after intra-arterial thrombolysis could result in subsequent thrombus formation and clinical deterioration. Glycoprotein IIb/ IIla inhibitors have been shown to reduce this elevation in procoagulants. We present a pilot study of three cases of acute basilar artery occlusion treated with a combination of intra-arterial thrombolytics and Gp IIb/IIla inhibitor with remarkable clinical recovery seen in all the patients.
\end{abstract}

Key words: Basilar artery thrombosis, glycoprotein Ilb/llla inhibitor, intra-arterial thrombolysis

\section{Introduction}

Basilar artery thrombosis is a serious condition and commonly affects the elderly with male preponderance. If untreated it is usually fatal in most cases or severely disabling. ${ }^{[1]}$ In acute basilar artery occlusion intra-arterial (IA) thrombolysis has been shown to be associated with good outcomes. ${ }^{[2-4]}$ Recent studies in acute myocardial infarction have demonstrated that thrombolytic and glycoprotein IIb/IIIa receptor inhibitor agents have a synergistic effect in achieving rapid and complete arterial recanalization without associated increase in severe hemorrhagic complication. ${ }^{[5-7]}$ Only few studies have reported a combination of systemic (intravenous) recombinant tissue plasminogen activator (tPA) and tirofiban, a nonpeptide glycoprotein IIb/IIIa inhibitor, in acute basilar artery thrombolysis. ${ }^{[8]}$ We describe our experience of treating three patients with acute basilar artery occlusion with a combination of tPA and abciximab given locally into the proximal thrombus interface intra-arterially.

\section{Case Reports}

\section{Case 1}

A 29-year-old male with no known risk factors presented with sudden onset vertigo, dizziness, hyperesthesia, rapid onset right hemiparesis, diplopia, and drowsiness. On neurological examination a admission, within three hours ollowing onset of stroke, he was in coma with bilateral complete ophthalmoplegia and right-side hemiplegia.

Noncontrast computerized tomography (NCCT) scan of the brain [Figure 1a] showed hyperdense thrombus in the basilar artery with no major infarcts. Magnetic resonance imaging (MRI) of the brain revealed an acute infarct in the midbrain, bilateral thalami, and the cerebellum with midbasilar artery occlusion [Figures $1 \mathrm{~b}$ and $\mathrm{c}$. Digital subtraction angiography (DSA), [Figures 1d and e] confirmed the findings seen on MR-angiography (MRA). Local IA thrombolysis was initiated with tPA (mixed as $1 \mathrm{mg}$ per milliliter 

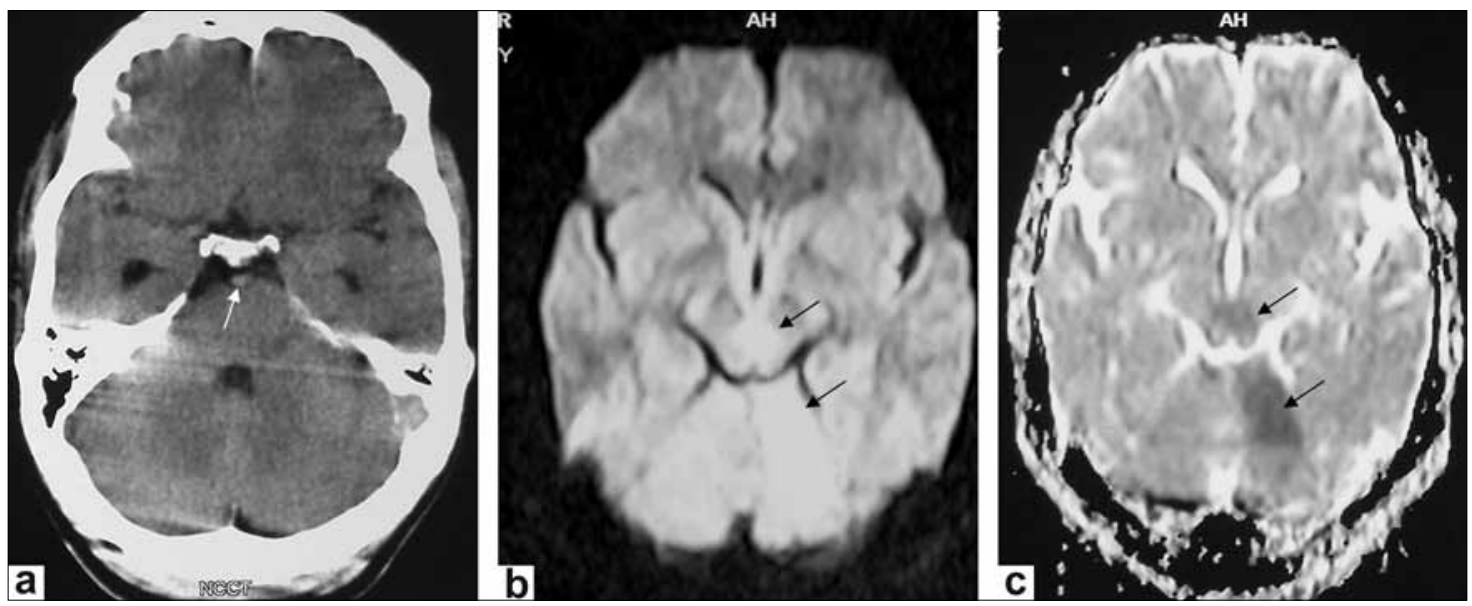

Figure 1: (a) Noncontrast CT scan of the brain (obtained at $7.30 \mathrm{am}$ ) showing hyperdense thrombus in the basilar artery with no major infarcts (arrow); (b,c) Magnetic resonance image of the brain (obtained at 8.05 am) revealing an acute infarct in the midbrain (arrow), bilateral thalami, and the cerebellum

saline) with small aliquots of $5 \mathrm{mg}$ per injection into the proximal thrombus interface. After IA application of a total dose of $20 \mathrm{mg}$, the final superselective angiogram revealed partial recanalization of the basilar artery. This was then followed by IA infusion of aliquots of $2 \mathrm{mg}$ each of abciximab till a dose $4 \mathrm{mg}$ was reached. Following which, there was complete recanalization of the basilar artery with good distal perfusion in the posterior circulation [Figures $1 \mathrm{f}$ and g]. Within six hours of thrombolysis, the patient started to improve. The follow-up CT scan revealed no hemorrhage. All investigations for cause of stroke were negative. At the time of discharge at one week he was alert with mild nondisabling incoordination in gait, bilateral mild ptosis, and left sixth nerve palsy. Follow up at three months showed only residual left sixth nerve palsy.

A repeat cerebral MRA [Figure 1h (pretreatment), I (post-treatment)] four weeks later showed sustainable patency of the basilar artery. He has had more than two years of clinical follow up and except for partial sixth nerve palsy he is fully recovered. He is on aspirin $150 \mathrm{mg}$ for secondary prevention.

\section{Case 2}

A 52-year-old man, a known hypertensive, presented with sudden onset vertigo with sweating and immediate unconsciousness. Three years before this illness he had left hemiparesis from which he improved completely. On evaluation, within five hours of stroke in the Emergency Department (ED), he was in coma and his left pupil was dilated and nonreacting with bilateral pyramidal signs.

Noncontrast computerized tomography of the brain was normal except for hyperdense thrombus in the basilar trunk [Figure 2a, arrow]. MRI brain with timeof- flight (TOF) MRA sequences revealed midbrain and bilateral thalamic infarcts and a distal basilar thrombus
[Figure 2b-d]. Superselective angiogram was performed and local (IA) infusion of $20 \mathrm{mg}$ of tPA was given. Check angiogram revealed a partial recanalization of the basilar artery [Figure 2e and f]. A total of $10 \mathrm{mg}$ of abciximab was given locally in small doses of $2 \mathrm{mg}$ each till a good antegrade flow was established. Subcutaneous low molecular weight heparin was given for a period of seven days following thrombolysis. Clinically, the patient improved remarkably within the next 48 hours. He became ambulant within a week of onset of stroke and was discharged on the fourteenth day with a modified Rankin score (mRS) of 2. A follow up MRI showed complete recanalization of the basilar artery with flow in both the posterior cerebral arteries [Figure $2 \mathrm{~g}$ and $\mathrm{h}$ ]. Investigations showed moderate left ventricular dysfunction and hypokinetic apex. He was presumed to have a cardioembolic event and was discharged on oral anticoagulants with an advice to maintain an INR of 2-2.5.

\section{Case}

A 48-year-old man presented with history of sudden onset of altered sensorium of four hours duration prior to presentation into the ED. There were no seizures or headache at onset. He had no known vascular risk factors except chronic smoking. On examination, he was responsive feebly to painful stimuli, his right pupilwas dilated with sluggish reaction to light, and there was a left facial palsy, right hemiplagia, and bilateral pyramidal signs.

Noncontrast computerized tomography brain showed left cerebellar infarct with hyperdense thrombus in the basilar trunk [Figure 3a, arrow]. Superselective angiography confirmed proximal basilar artery thrombosis and nonfilling of the entire basilar artery suggesting a long column of thrombus and a large thrombus burden [Figure 3b]. Owing to the proximal occlusion in the basilar artery and also due to the 

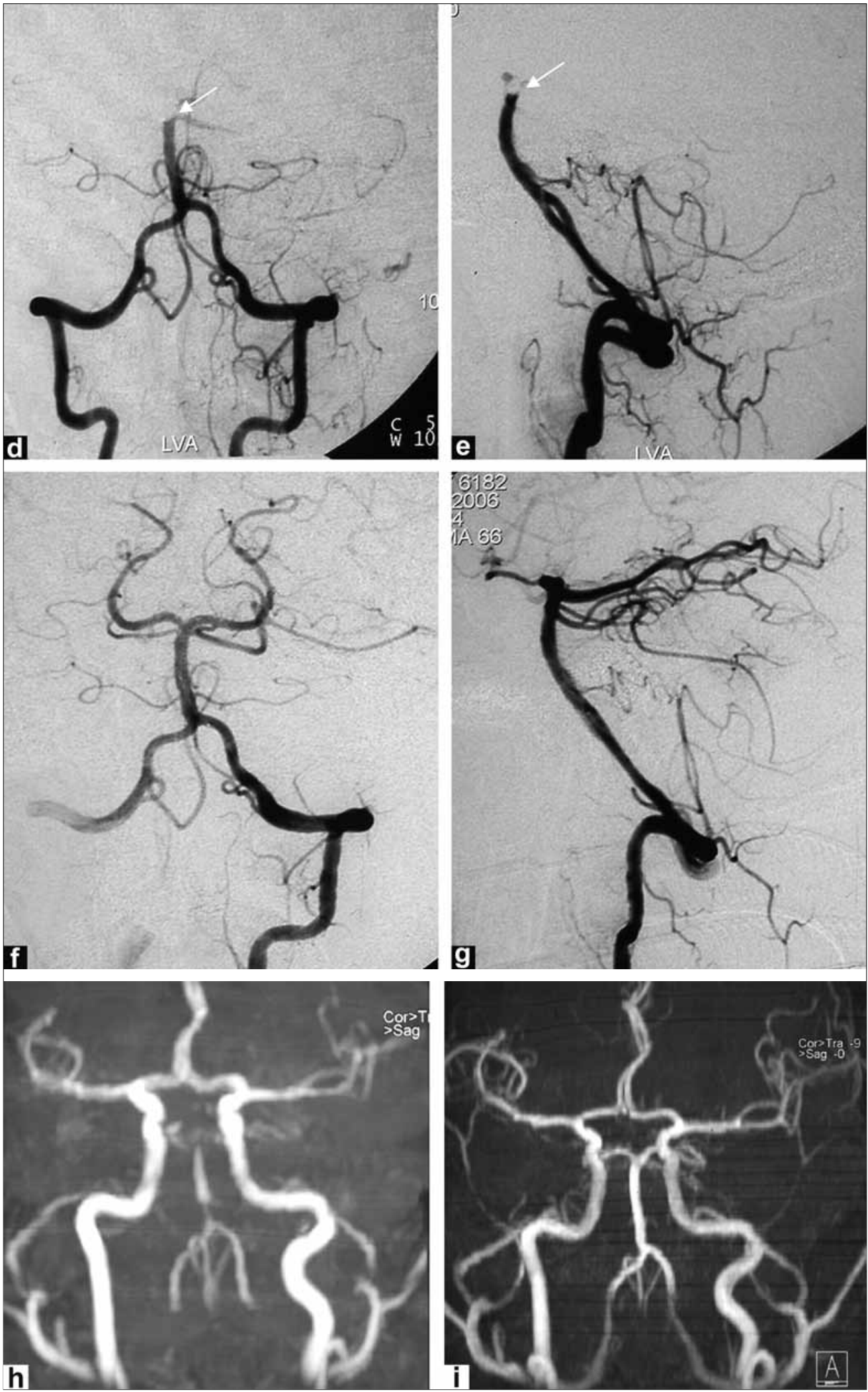

Figure 1: (d, e) Digital subtraction angiography (obtained at $9.01 \mathrm{am}$ ), confirming the occlusion of upper basilar trunk with nonfilling of the bilateral PCAs; $(f, g)$ Complete recanalization of the basilar artery with good distal perfusion in the posterior circulation (procedure over by 10.27 am); (h) Pretreatment MRA time of flight sequence showing occlusion of the distal basilar artery; (i) Follow up MRA obtained four weeks later showing sustained patency of the basilar artery 

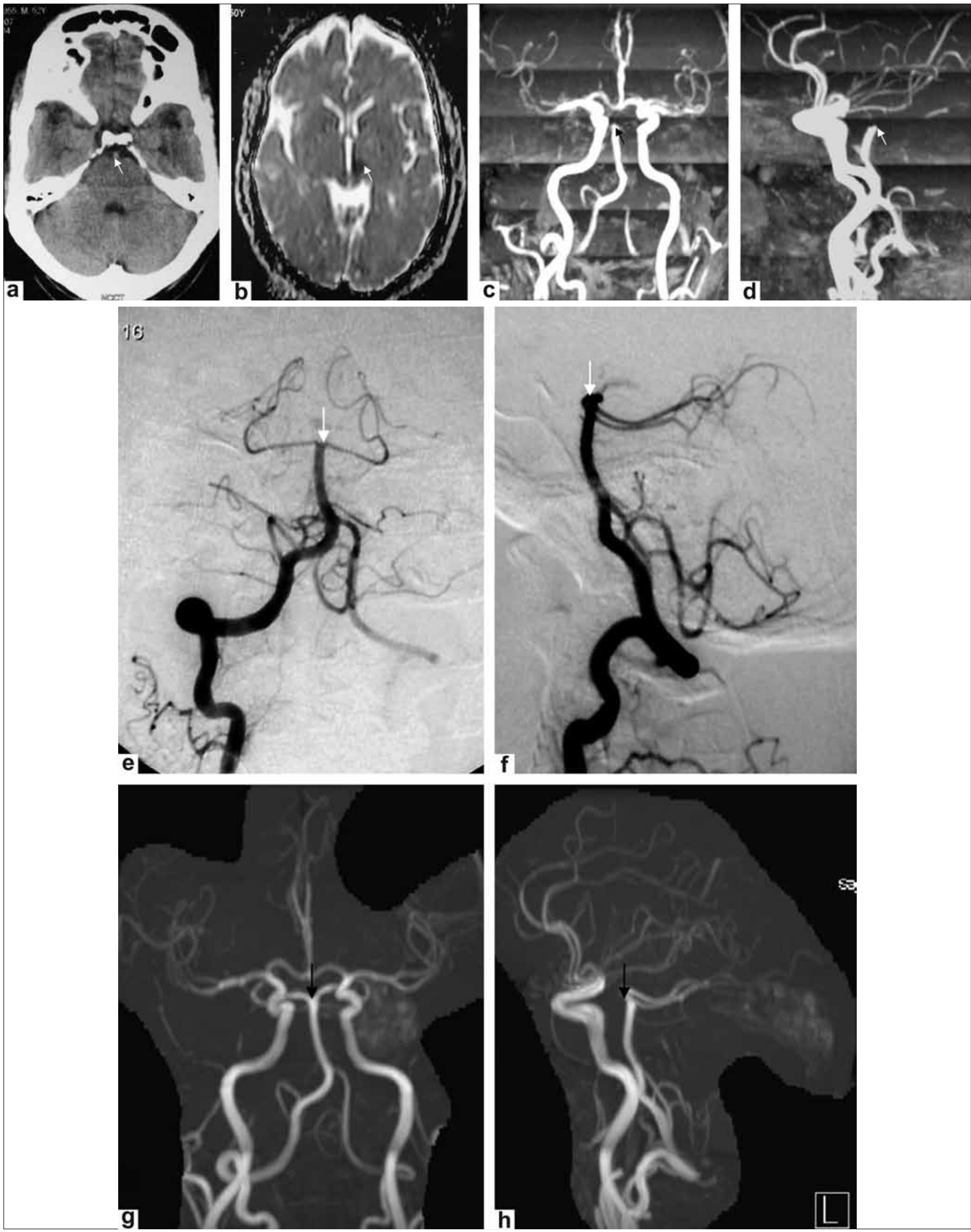

Figure 2: (a) Unenhanced CT (7.50 am) showing hyperdense thrombus in the basilar trunk without parenchymal infarcts (arrow); (b, c, d) MRI brain ADC map with time of flight MRA sequences revealing midbrain and bilateral thalamic infarcts and distal basilar thrombus (9.05 am); (e, $f$ ) Magnetic resonance angiogram showing occlusion of basilar top with preserved flow into both superior cerebellar arteries $(10.10 \mathrm{am}) ;(\mathrm{g}, \mathrm{h})$ Magnetic resonance angiogram obtained four weeks later showing recanalized basilar top with good flow into the posterior cerebral arteries 
preferential flow into the dominant right posterior inferior cerebellar artery (PICA), it gave an impression that right vertebral artery (VA) is ending in PICA [Figure 3b]. Tissue plasminogen activator was infused (total dose of $20 \mathrm{mg}$ ) followed by $10 \mathrm{mg}$ of abciximab with establishment of good antegrade flow up to the distal basilar artery [Figure 3c]. Retrograde filling of upper basilar trunk was observed on selective injection of internal carotid artery [Figure 3d, arrow].

Owing to the large thrombus burden, partial and significant recanalization could be achieved within the limited doses of the combined thrombolytics and antiplatelet. Since good collateral flow was noticed in the retrograde direction [Figure 3d, arrow] with opacification of the upper basilar, wedecided to stop at this moment to prevent from exceeding the dose limits to avoid hemorrhagic complications. Post thrombolysis, the patient's sensorium improved over the next 24 hours. His hemiparesis also improved but remained ataxic. At the time of discharge he could walk with support but continued to have combined vertical and horizontal gaze palsy with left skew and vertical diplopia. The right pupil was still dilated and nonreactive to light. One month after discharge, the patient's horizontal gaze palsy completely resolved. MRA obtained 18 days later showed complete recanalization of the artery [Figure 3e].

A complete recanalization of the occluded artery on follow up MRA 18 days after the procedure proves the often stated point that tPA does have a continued
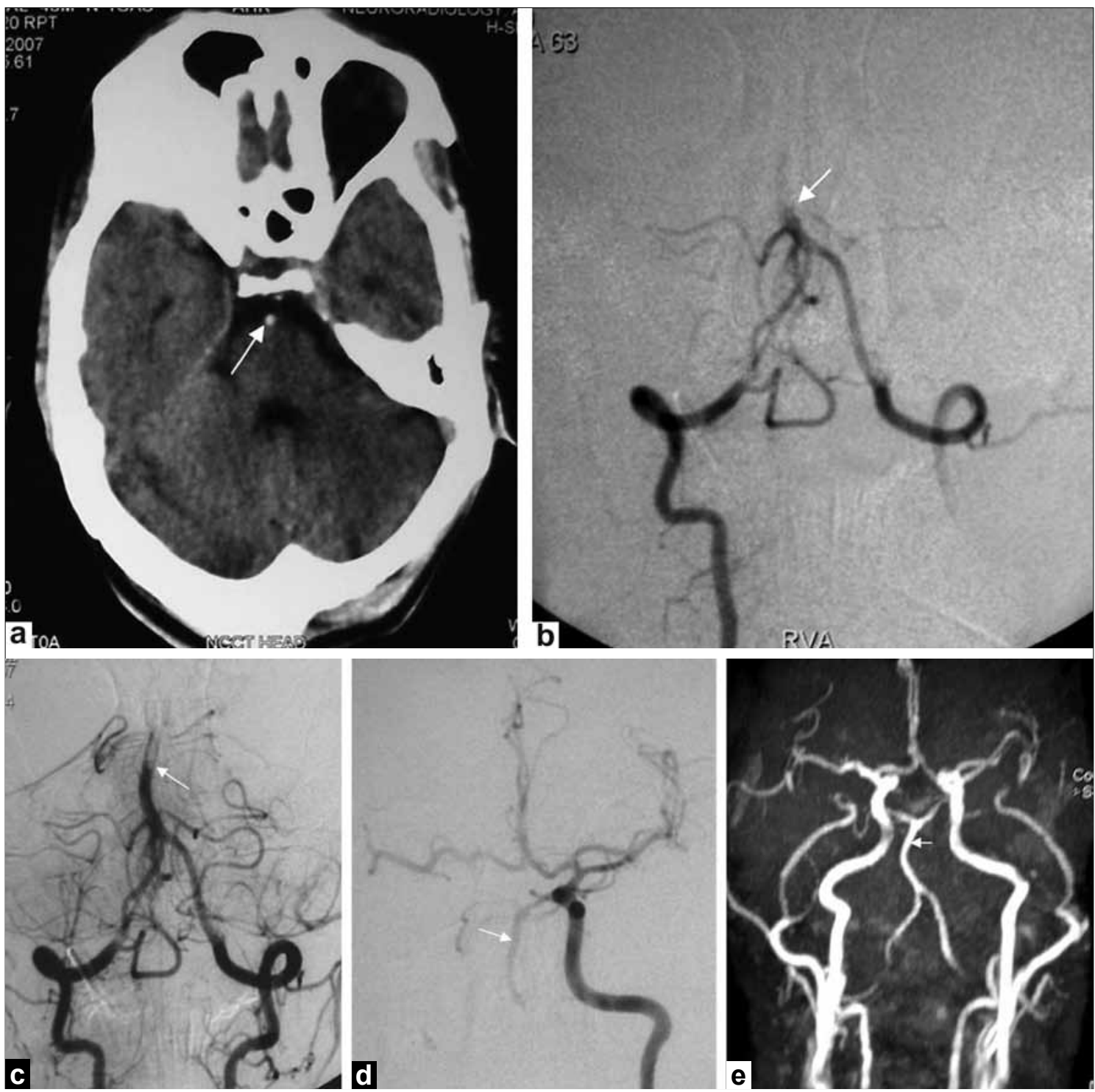

Figure 3: (a) Noncontrast CT of the head (10.54 am) showing left cerebellar infarct with hyperdense thrombus in the basilar trunk; (b) Superselective angiography $(\mathbf{1 2 . 5 0} \mathrm{pm})$ of the right vertebral artery confirming proximal basilar artery thrombosis. The PICA on right side is also dominant with good filling owing to the distal occlusion; (c) There is partial recanalization of the artery of the rtPA and abciximab infusion through microcatheter; microcatheter tip was placed in the mid basilar portion beyond the origin of ipsilateral PICA (1.20 pm); (d) Retrograde filling of upper basilar trunk (arrow) observed on selective injection of internal carotid artery after the infusion therapy was over (1.25 pm); (e) Magnetic resonance angiogram obtained 18 days later showing complete recanalization of the artery 
and sustained action once it binds with the thrombus following its delivering into the thrombus through the microcatheter. The microcatheter tip was placed in the mid portion of the thrombus which enabled us to infuse the tPA in the midbasilar segment well beyond the origin of the right PICA. This also ensured effective and targeted delivery of the drug.

\section{Discussion}

Review of the literature found very few case studies reporting the combination therapy. ${ }^{[8]}$ Junghans and colleagues were the first group to report the use of IA tPA with intravenous tirofiban for treating acute basilar artery thrombosis. They treated four patients with this combination and found rapid and sustained clinical improvement in all and also demonstrated patency of the intracranial vessels. ${ }^{[8]}$

Intra-arterial thrombolysis has been shown to be as effective or superior to intravenous thrombolysis in lysing the clot as well as improving patient outcome. ${ }^{[9,10]}$ It has been documented that markers of procoagulant activity may increase tremendously immediate posttreatment with tPA (peaks after 1-3 hours and persists for up to 72 hours), indicating that thrombolysis may trigger the coagulation cascade and hence further enhance thrombin formation at the site of occlusion ${ }^{[11]}$. This may be one explanation for recanalization failures seen at times with $\mathrm{PPA}$ administration.

The role of glycoprotein IIb/IIIa receptors and their role in the coagulation cascade have been well elucidated. ${ }^{[2]}$ Activated glycoprotein $\mathrm{IIb} / \mathrm{III}$ a receptor binds fibrinogen molecules, which form bridges between adjacent platelets thereby facilitating platelet aggregation and accumulation. Thrombin increases fibrin deposition favoring incorporation of platelets into the thrombus in the downstream vascular territories and may obscure heparin-binding sites impeding heparin to prevent reocclusion. Thus, glycoprotein IIb/IIIa inhibitors inhibit this aggregation of platelets which occurs secondary to a successful thrombolysis.

Selective catheterization of the target vessel along with administration of thrombolytic agents into the thrombus is now considered most optimal for faster, more complete recanalization of the acute basilar occlusion. Complete reperfusion of the posterior circulation is taken as a conditional sine qua non for most favorable stroke outcome after basilar artery occlusion. Although IA thrombolysis has been proven optimal, this procedure requires expertise and adequate infrastructure which makes it amenable only to few selected centers. With this in view, some case series have also been reported using intravenous delivery of the fibrinolytic agent and glycoprotein IIb/IIIa receptor blocker as infusion over several hours. Alex Abou-Chebl et al. ${ }^{[13]}$ have suggested a multimodal therapy for severe ischemic stroke combining GpIIb/IIIa antagonists and angioplasty after failure of thrombolysis.

All the three patients treated within 6-8 hours of onset of stroke with combination of fibrinolysis and glycoprotein $\mathrm{IIb} / \mathrm{III}$ a receptor blocker have done extremely well with outcome of mRS less than 2 . No symptomatic or asymptomatic cerebral or extracerebral hemorrhagic complications were observed. None of the patients developed thrombocytopenia, known to occur with glycoprotein $\mathrm{IIb} / \mathrm{III}$ a receptor inhibitor administration. It is well known that abciximab can cause thrombocytopenia if used in larger doses and for prolonged period. Knowing this fact fully, we decided to use small doses of IA infusion to maximize the drug effectiveness and offset its potential adverse effects. To have sustained antiplatelet action all our patients were put on aspirin $150 \mathrm{mg}$ per day (life-long) and clopidogrel for the first three months. Regular blood tests were carried out to detect any suggestion of thrombocytopenia.

Though our cohort was small, only three patients, we found that a combination of small doses $(8-10 \mathrm{mg})$ of abciximab and tPA administered intra-arterially resulted in rapid and sustained recanalization of the basilar artery with rapid improvement in the clinical condition of the patient without significant hemorrhagic complication. We further wish to indicate that the combination of rtPA and antiplatelet is investigational at the moment and we do not wish to draw any meaningful conclusions from our small pilot study.

\section{References}

1. Archer CR, Horenstein S. Basilar artery occlusion: Clinical and radiological correlation. Stroke 1977;8:383-90.

2. Zeumer H, Hacke W, Ringelstein EB. Local intra-arterial thrombolysis in vertebro-basilar thromboembolic disease. AJNR Am J Neuroradiol 1983;4:401-4.

3. Hacke W, Zeumer H, Ferbert A, Bruckmann H, del Zoppo GJ. Intraarterial thrombolytic therapy improves outcome in patients with acute vertebrobasilar occlusive disease. Stroke 1988;19:1216-22.

4. Zeumer H, Freitag HJ, Grzyska U, Neunzig HP. Local intra-arterial fibrinolysis in acute vertebrobasilar occlusion: Technical developments and recent results. Neuroradiology 1989;31:336-40.

5. Ohman EM, Kleiman NS, Gacioch G, Worley SJ, Navetta FI, Talley JD, et al. Combined accelerated tissue-plasminogen activator and platelet glycoprotein IIb/IIIa integrin receptor blocked with Integrilin in acute myocardial infarction: Results of a randomized, placebo-controlled, doseranging trial. IMPACT-AMI Investigators. Circulation 1997;95:846-54

6. Antman EM, Giugliano RP, Gibson CM, McCabe CH, Coussement $\mathrm{P}$, Kleiman NS, et al. Abciximab facilitates the rate and extent of thrombolysis: Results of the thrombolysis in myocardial infarction (TIMI) 14 trials. The TIMI 14 Investigators. Circulation 1999;99: 2720-32.

7. Kuker W, Friese S, Vogel W, Schmidt F, Weller M. Incomplete resolution of basilar artery occlusion after intra-arterial thrombolysis: Abciximab 
and heparin prevent early rethrombosis. Cerebrovasc Dis 2000;10:484-6.

8. Junghans U, Seitz RJ, Wittsack HJ, Aulich A, Siebler M. Treatment of acute Basilar thrombosis with a combination of systemic alteplase and tirofiban a non-peptide glycoprotein IIb/IIIa inhibitor: Report of four cases. Radiology 2001;221:795-801.

9. Becker K, Monsein L, Ulatowski J, Mirski M, Williams M, Hanley DF. Intraarterial thrombolysis in vertebrobasilar occlusion. AJNR Am J Neuroradiol 1996;17:255-62.

10. Qureshi AI, Boulos AS, Hanel RA, Suri MF, Yahia AM, Alberico RA, et al. Randomized comparison of intra-arterial and intravenous thrombolysis in a canine model of acute basilar artery thrombosis. Neuroradiology 2004;46:988-95.

11. Fassbender K. Dempfle CE, Mielke O, Schwartz A, Daffertshofer M,
Eschenfelder C, et al. Changes in coagulation and fibrinolysis markers in acute ischemic stroke treated with recombinant tissue plasminogen activator. Stroke 1999;30:2101-4.

12. Lefkovits J, Plow EF, Topol EJ. Platelet glycoprotein IIb/IIIa receptors in cardiovascular medicine. N Engl J Med 1995;332:1553-9.

13. Abou-Chebl A, Bajzer CT, Krieger DW, Furlan AJ, Yadav JS. Multimodal therapy for the treatment of severe ischemic stroke combining GPIIb/IIIa antagonists and angioplasty after failure of thrombolysis. Stroke 2005;36:2286-8.

Accepted on 31-03-2009

Source of Support: Nil, Conflict of Interest: None declared. 